\title{
Focal Electrical Stimulation of Human Retinal Ganglion Cells
}

\author{
Sasi Madugula* 3,4,7, Alex R. Gogliettino*3,7, Moosa Zaidi 1,4,7, Gorish Aggarwal 1,2,7, Alexandra \\ Kling 1,7, Nishal P. Shah 1,2,7, Ramandeep Vilkhu 2, Maddy Hays 7,8, Huy Nguyen 1, \\ Victoria Fan 1, Eric G. Wu 2,7, Pawel Hottowy ${ }^{9}$, Alexander Sher 5, Alan M. Litke ${ }^{5}$, \\ Ruwan A. Silva 6, E.J. Chichilnisky 1,6,7 \\ 1. Department of Neurosurgery, Stanford University, Stanford, CA, USA \\ 2. Department of Electrical Engineering, Stanford University, Stanford, CA, USA \\ 3. Neurosciences PhD Program, Stanford University, Stanford, CA, USA \\ 4. School of Medicine, Stanford University, Stanford, CA, USA \\ 5. Santa Cruz Institute for Particle Physics, University of California, Santa Cruz, CA, USA \\ 6. Department of Ophthalmology, Stanford University, Stanford, CA, USA \\ 7. Hansen Experimental Physics Laboratory, Stanford University, Stanford, CA, USA \\ 8. Department of Bioengineering, Stanford University, Stanford, CA, USA \\ 9. Faculty of Physics and Applied Computer Science, AGH University of Science and Technology, \\ Krakow, Poland \\ * equal contributions
}

\begin{abstract}
Electrical stimulation of retinal ganglion cells (RGCs), which transmit visual information to the brain, is used in retinal implants to treat blindness caused by photoreceptor degeneration. However, the performance of existing clinical implants is limited by indiscriminate stimulation of many cells and cell types. Recent work in isolated macaque retina has shown the ability to precisely evoke spikes in the major RGC types by direct electrical stimulation at safe current levels, with single-cell, single-spike resolution and avoidance of axon bundle activation in many cases. However, these findings have not been verified in the human retina. Here, electrical activation of the major human RGC types was examined using large-scale, multi-electrode recording and stimulation and compared to results from several macaque retinas obtained using the same methods. Electrical stimulation of the major human RGC types closely paralleled results in macaque, with similar somatic and axonal stimulation thresholds, cellular and cell type selectivity of stimulation, avoidance of axon bundle stimulation by calibration, targeting of different cell types based on their distinct electrical signatures, and potential efficacy of real-time stimulus optimization for artificial vision. The results indicate that the macaque retina provides a quantitatively accurate picture of how focal electrical stimulation can be used in future highresolution implants.
\end{abstract}

Acknowledgements: The human eye was provided by Donor Network West (San Ramon, CA). We are thankful for the cooperation of Donor Network West and all of the organ and tissue donors and their families, for giving the gift of life and the gift of knowledge, by their generous donations. We thank T. Moore, M. Taffe, K. Bankiewicz, J. Carmena, W. Newsome and the California National Primate Research Center for access to macaque retinas. R. Samarakoon and S. Kachiguine for technical assistance. We thank S. Mitra for valuable discussion and ideas. Support: Pew Charitable Trust Scholarship in the Biomedical Sciences (AS), a donation from John Chen (AML), Polish National Science Centre grant DEC-2013/10/M/NZ4/00268 (PH), Research to Prevent Blindness Stein Innovation Award, Wu Tsai Neurosciences Institute Big Ideas, NIH NEI R01-EY021271, NIH NEI R01-EY029247, and NIH NEI P30EY019005 (EJC).

Contributions: SM and AK performed dissections and collected the data, SM, ARG and EJC conceived and designed the experiments, SM, ARG, RV, MZ, GA, NPS, MH, VF, HN, EGW, and EJC analyzed the data, $\mathrm{PH}, \mathrm{AS}$ and $\mathrm{AML}$ developed and supported recording hardware and software, RAS performed the surgery, EJC supervised the project. EJC, SM, ARG, MZ, NPS, GA, and RV wrote the paper. 


\section{Introduction}

Direct electrical stimulation of retinal ganglion cells (RGCs) can be used to deliver artificial visual signals to the brain, and has been harnessed by retinal implants to restore vision in people blinded by photoreceptor degeneration [1, 2]. Unfortunately, clinical devices based on this approach provide limited restoration of visual function due to indiscriminate activation of many cells and cell types [2]. This suggests that stimulation of earlier retinal neurons -specifically, bipolar cells -- may be more effective for sight restoration [3, 4], despite the limited scientific understanding of bipolar cells and the difficulties of recording from them to validate stimulation. However, extensive experiments in isolated macaque monkey retina have demonstrated that RGCs can be activated precisely by direct electrical stimulation at very low current levels, in many cases with single-cell, single-spike, cell-type resolution [5-9], with the potential to deliver high-quality artificial vision [10,11]. Because electrical stimulation and recording have never been performed simultaneously in the human retina, it remains unclear whether these promising findings in macaque retina can be used to develop high-resolution retinal implants for vision restoration.

Here, large-scale multi-electrode stimulation and recording from hundreds of RGCs, combined with cell-type identification using visual stimulation, were applied to isolated healthy human retina, and the findings were compared to results from dozens of macaque retinas obtained in the same experimental conditions $[5,6,9,10]$. The results revealed a striking similarity between the properties of human and macaque retina, including low electrical stimulation thresholds for the major cell types, selectivity of activation, separability of cell types based on their electrical properties, and potential efficacy of real-time stimulus optimization for artificial vision. These results extend the body of work in macaque retina and support the use of this animal model for the development of high-resolution retinal implants in treating vision loss.

\section{Results}

To probe the focal electrical activation of the major human RGC types, simultaneous stimulation and recording with a large-scale high-density multi-electrode array system (512 electrodes, 60 $\mu \mathrm{m}$ pitch), combined with visual stimulation, were performed in an isolated human retina using procedures developed for macaque retina (see Methods). To identify the four major RGC types in the human retina, white noise visual stimulation was performed, and reverse correlation was used to summarize the spatiotemporal receptive field properties of each cell [12-14]. These properties, and the mosaic spatial organization of functionally identified cell types (Fig. 1, side panels), uniquely identified the ON-parasol, OFF-parasol, ON-midget and OFF-midget RGC types [13-15]. These are the numerically dominant RGC types in the human and non-human primate retina, accounting for $\sim 65 \%$ of the entire visual signal transmitted to the brain (see [16, $17])$. In this study, analysis was restricted to these four cell types. 

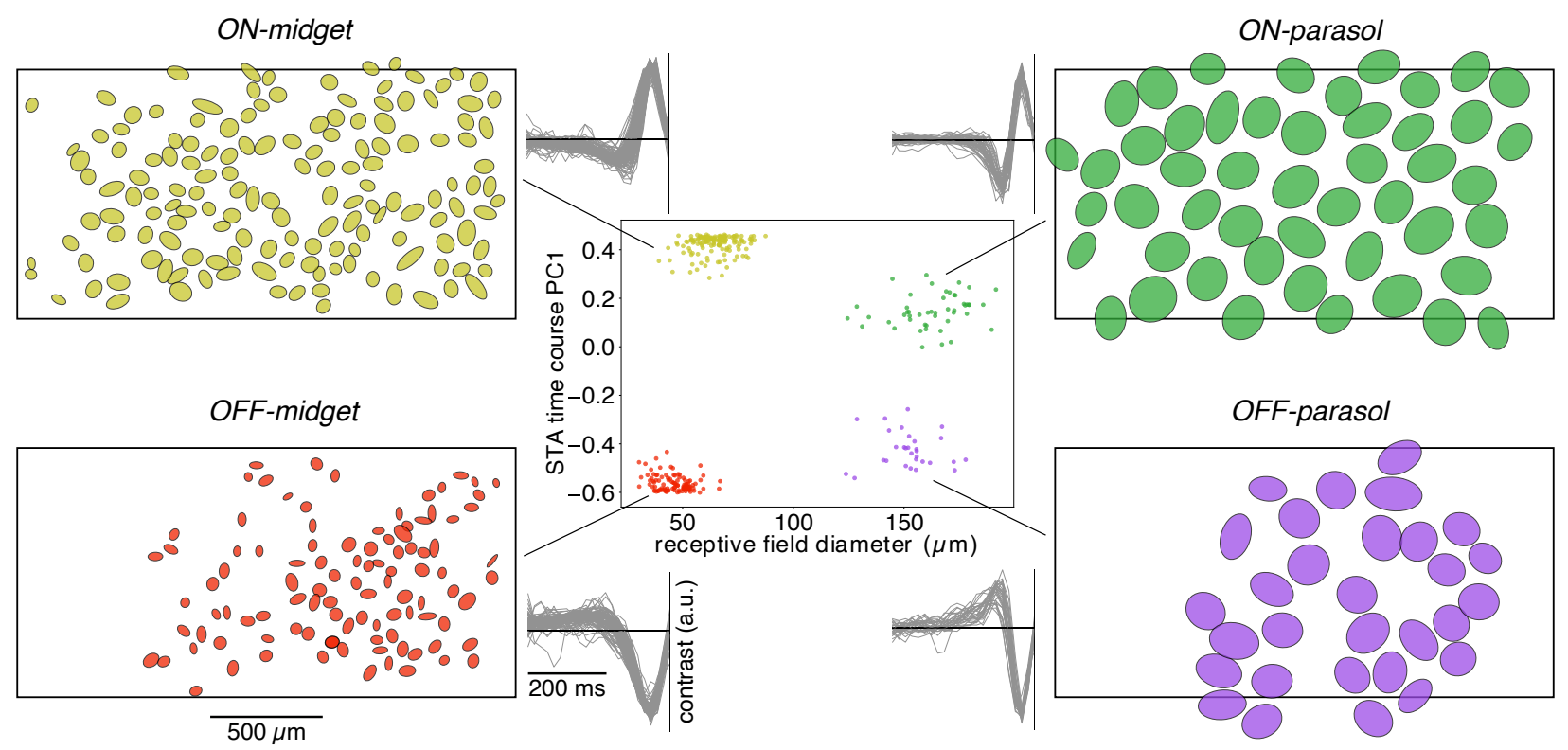

Figure 1. Cell type classification. Center: Recorded cells were separated into distinct functional clusters by examining the receptive field diameter and the projection onto the first principal component of light response time courses obtained from the spike-triggered average (STA) stimulus. The four numericallydominant cell types in the human retina -- ON-parasol, OFF-parasol, ON-midget, and OFF-midget -- were identified from these clusters. Side panels: receptive field mosaics of each cell type and overlaid STA time courses of all cells from each cluster. The black rectangular outline represents the approximate location of the electrode array.

A brief $(\sim 0.1 \mathrm{~ms})$ weak $(\sim 1 \mu \mathrm{A})$ pulse of current delivered extracellularly through a single electrode was able to evoke a single spike in one or more human RGCs with sub-millisecond temporal precision. To quantify the probabilistic firing of a RGC in response to electrical stimulation, a triphasic current pulse (Fig. 2A\&D, top inset) was delivered repeatedly through a single electrode. In the $2 \mathrm{~ms}$ time window after the current pulse, the recorded voltage waveform on the stimulating electrode and nearby electrodes during a given stimulation trial generally took one of two forms: electrical artifact alone, or electrical artifact with an evoked spike superimposed (Fig. 2A\&D, bottom inset). To separate the evoked spike waveform from electrical artifact, the average of the artifact-only traces was subtracted from each evoked spike trace. Each evoked spike waveform was then assigned to the cell with the most similar spike waveform recorded during light stimulation (Fig. 2A\&D, see Methods). For a given cell, the variation in the time of the evoked spike from trial to trial was small (0.03 ms SD; Fig. 2B\&E), as expected with directly electrically evoked spikes [18]. Increases in current amplitude produced a progressively greater probability of evoking a spike on a given trial, a relationship that was summarized by a sigmoidal activation curve (Fig. 2C\&F). The activation threshold was defined as the current level that produced an interpolated spike probability of 0.5 . 

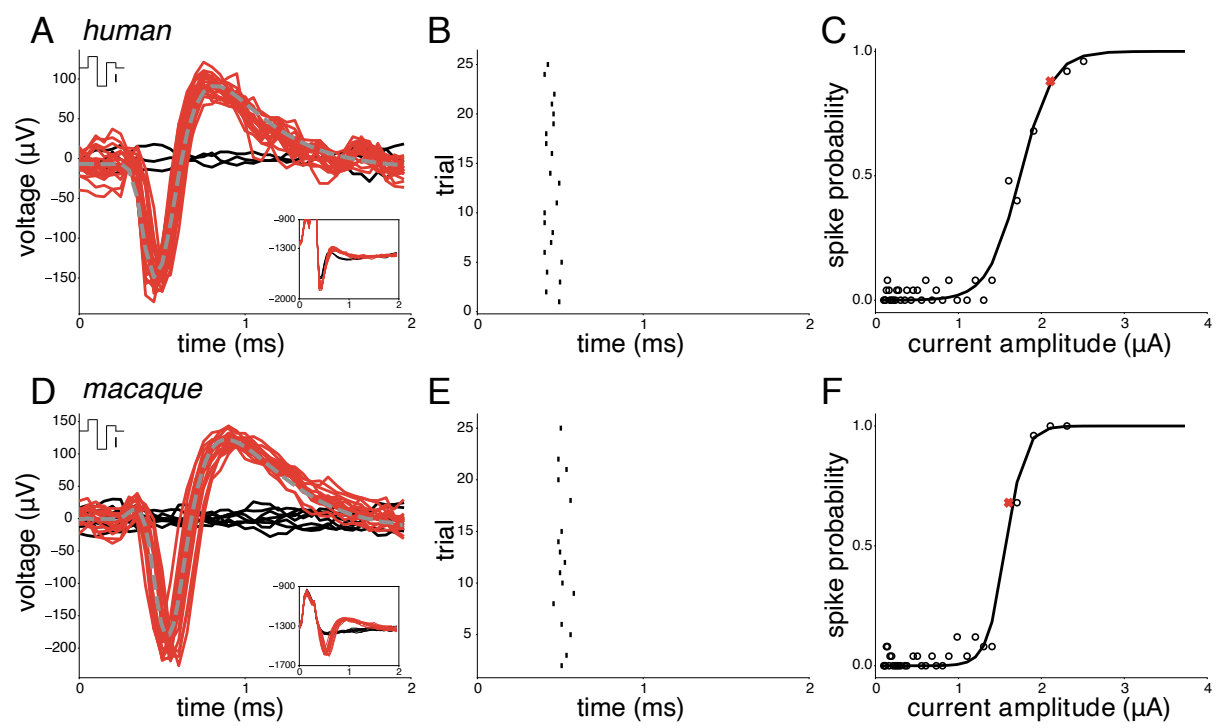

Figure 2. Spike sorting of electrical stimulation data for human (A-C) and macaque (D-F) RGCs. A,D. Artifact-subtracted voltage traces (red, black) recorded after 25 trials of stimulation with a triphasic current pulse (top inset, scale bar: $1 \mu \mathrm{A}$ ). The recorded waveforms on many trials (red) resembled the average spike waveform obtained without electrical stimulation (gray, dashed). Other trials resulted in no spikes (black). The artifact estimate was obtained by averaging the responses recorded in non-spiking trials (black, inset). Substantial differences were observed in the electrical artifact waveform between electrodes and experiments. B,E. Latency of electrically-elicited spikes (tick marks) from trial to trial. C,F. Response probability as a function of current amplitude. For each amplitude, the response probability (open circles) was computed, and a sigmoidal curve was fitted to the results (black curve, see Methods). The red " $X$ " indicates the current level used in the previous panels.

This spike sorting procedure was used to compute activation curves at multiple stimulating electrodes, to obtain spatial maps of the sensitivity to electrical stimulation for each recorded cell (Fig. 3A, middle). To select electrodes for analysis, average spike waveforms recorded in the absence of electrical stimulation were used to identify somas and axons, based on their amplitude, biphasic or triphasic temporal structure, and propagation over space (Fig. 3A, top; [19]). These features broadly resembled the spatiotemporal spike waveforms observed in macaque RGCs (Fig. 3B, top). The spatial maps revealed that each RGC could be activated at several locations on the soma and axon (Fig. 3A\&B).

To compare electrical response characteristics in different cell types, 66 activation thresholds resulting from somatic and axonal stimulation were examined in the four major human RGC types. Thresholds for all four types were in a similar range $(\sim 0.8-3.5 \mu \mathrm{A})$, regardless of whether the stimulating electrode was located over the soma or axon (Fig 4A). Activation thresholds aggregated over hundreds of RGCs from 13 macaque recordings overlapped substantially with those of human RGCs (Fig. 4A\&B; [6]). 

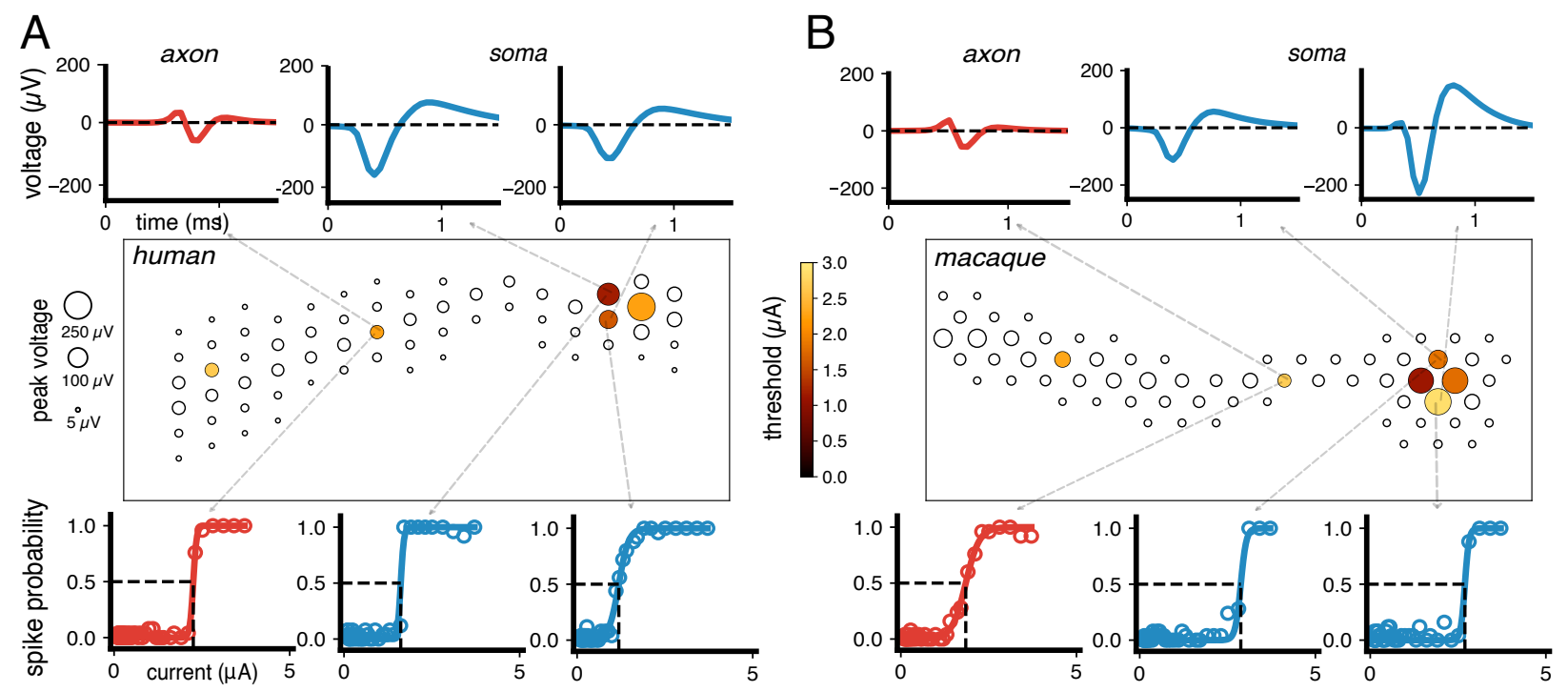

Figure 3. Electrical stimulation and recording of individual ON-parasol cells in the human $(A)$ and macaque $(B)$ retina. The electrical image $(E I)$ of each cell is displayed in a box representing a region of the electrode array, with circles indicating the locations of electrodes on which spikes from the cell were detected. The size of each circle represents the peak amplitude of the recorded spike at that electrode. Recorded spike waveforms are shown for three example electrodes located near the soma (top, blue) and axon (top, red). For each cell, several electrodes (filled circles) were capable of eliciting spikes with electrical stimulation in the range of current amplitudes tested, while others were not (open circles). The colors of the filled circles represent activation thresholds. Activation curves are shown for each example somatic (bottom, blue) and axonal (bottom, red) recording site. The threshold response probability and current are indicated by dashed lines (bottom).

To test whether an individual human RGC could be selectively activated without activating other nearby cells, the activation curves of a collection of RGCs in a small area were determined in response to current passed through a single electrode (Fig. 5). For each target cell tested, adjacent non-target cells were identified based on the proximity of their receptive fields, as a proxy for the proximity of their somas [20]. To examine selectivity within a cell type, the activation curve of the target cell was compared to the activation curves of the adjacent cells in the mosaic of the same type (Fig. 5A). Note that spike sorting could only be performed reliably in a subset (43/316) of the cells identified with visual stimulation (see Methods), limiting investigation to one or two small regions of the retina that contained locally complete mosaics of each cell type. Examples of target cell activation at current amplitudes that did not activate any of the neighboring non-target RGCs of the same type were identified for each of the four cell types (Fig. 5B). To determine whether selectivity across cell types could be achieved, a target OFF-midget cell and RGCs of all four major types with overlapping or immediately adjacent receptive fields were analyzed. Selective activation of the target OFF-midget cell was achieved without activating nearby cells of the other major types (Fig. $5 \mathrm{C}$ ). Although this analysis was performed only for a target OFF-midget cell in the present work, the results recalled previous findings for each of the major cell types in the macaque [6]. 


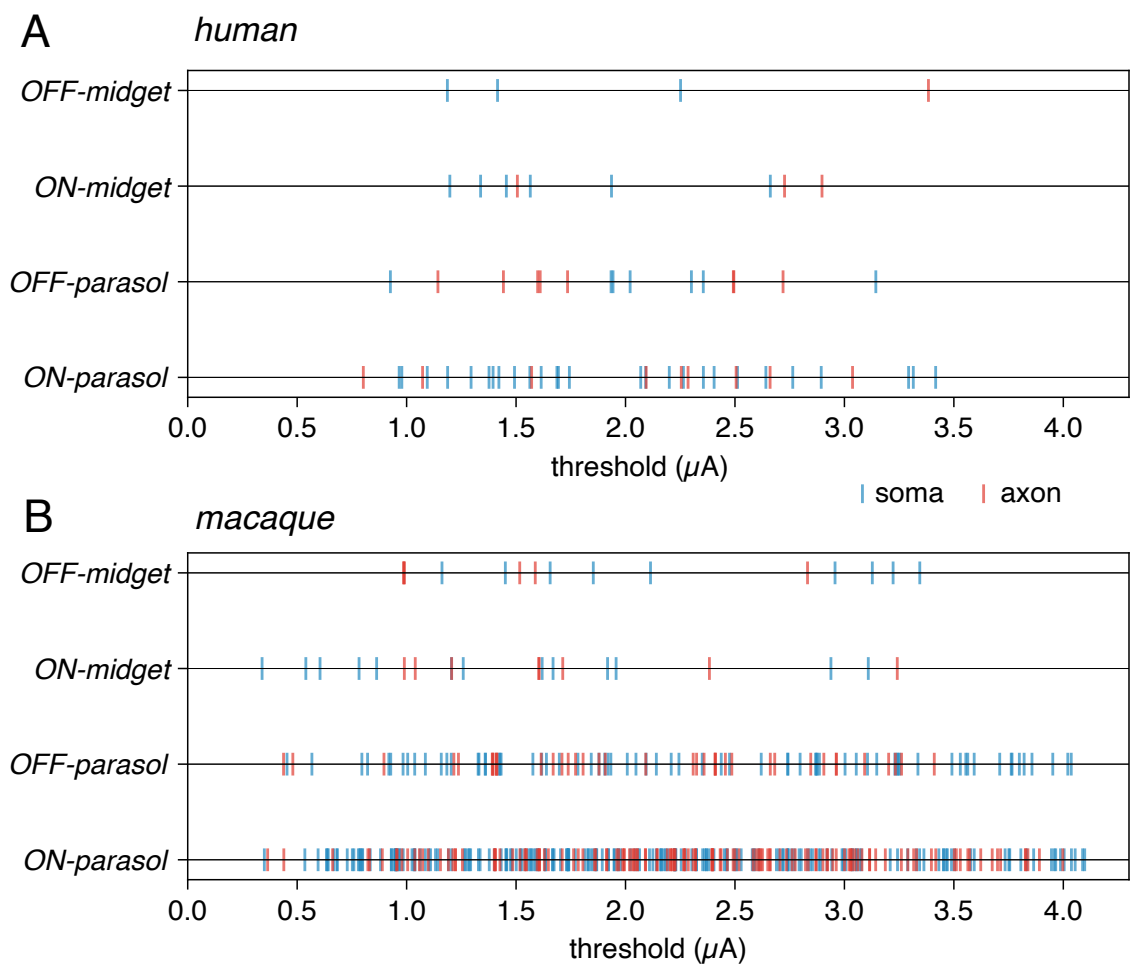

Figure 4. Electrical activation thresholds for the four numerically-dominant RGC types in human and macaque. A. Activation thresholds for ON and OFF parasol and ON and OFF midget cells from a single human retina recording (66 thresholds from 43 cells). Thresholds from stimulating electrodes located near somas and axons (see Fig. 3) are indicated in blue and red, respectively. B. Same as panel A, but for 13 macaque recordings (313 thresholds from 297 cells).

Reconstituting high-resolution vision requires not only selective activation of individual RGCs in local regions, but also avoiding axons that run in bundles across the retinal surface and convey visual signals from distant, unidentified cells. To test whether this was possible, bundle activation thresholds were measured at each electrode by identifying the lowest current amplitude that produced bi-directionally propagating electrical activity [9] that reached opposite edges of the array, instead of terminating at a soma on the array [21]. Bundle activation thresholds were then compared to RGC activation thresholds on the same electrodes. Most electrodes activated bundles at current levels below the activation thresholds of nearby RGCs (Fig. 6A), confirming the substantial challenge of bundle activation (see Discussion). However, $14 / 43$ cells (among three of the four major types) were activated below bundle threshold at 17/66 stimulation sites tested. Comparison to data from 13 macaque preparations revealed a similar distribution of RGC and bundle activation thresholds (Fig. 6B), and 103/512 cells were activated at 114/554 stimulation sites without bundle activation. In summary, although undesirable axon bundle activation limits the selectivity of epiretinal stimulation, with appropriate calibration the electrodes that activate individual RGCs at current levels below bundle threshold can be identified and used (see Discussion). 


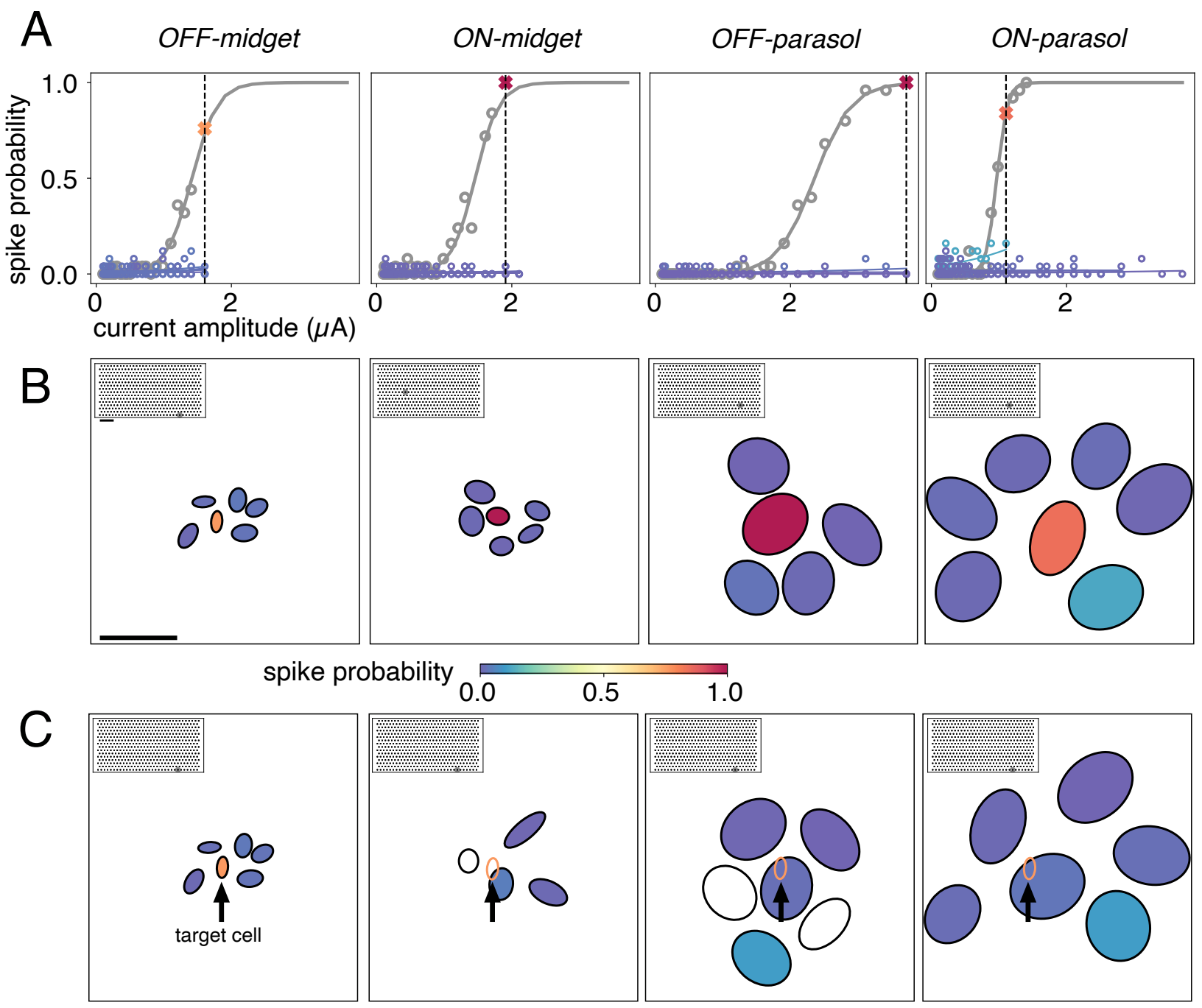

Figure 5. Cellular selectivity of electrical stimulation within and across cell types. A. Activation curves of a target cell (gray) and neighboring cells of the same type (shades of blue). For each cell type, it was possible to activate a target cell (spike probability $>0.75$ ) without activating neighboring cells of the same type (spike probability < 0.25 ). The largest current amplitude that produced selective activation is shown by the dashed vertical line, and the response probability of the target cell at this amplitude is marked by a red or orange "X". B. Receptive fields of the target cell (center) and neighboring cells from panel A. Receptive field fill colors indicate the spike probability at the current amplitude marked in panel A (vertical line). For each set of analyzed cells, the stimulating electrode location is represented by a gray dot on the inset array (scale bars: $250 \mu \mathrm{m}$ ). C. Same as panel B, but for a single OFF-midget target cell (arrow) with neighbors of each type shown in a separate panel. In the rightmost three panels, the target OFF-midget cell receptive field is represented by an open orange ellipse. The open black receptive fields indicate cells for which spikes could not be reliably identified. 

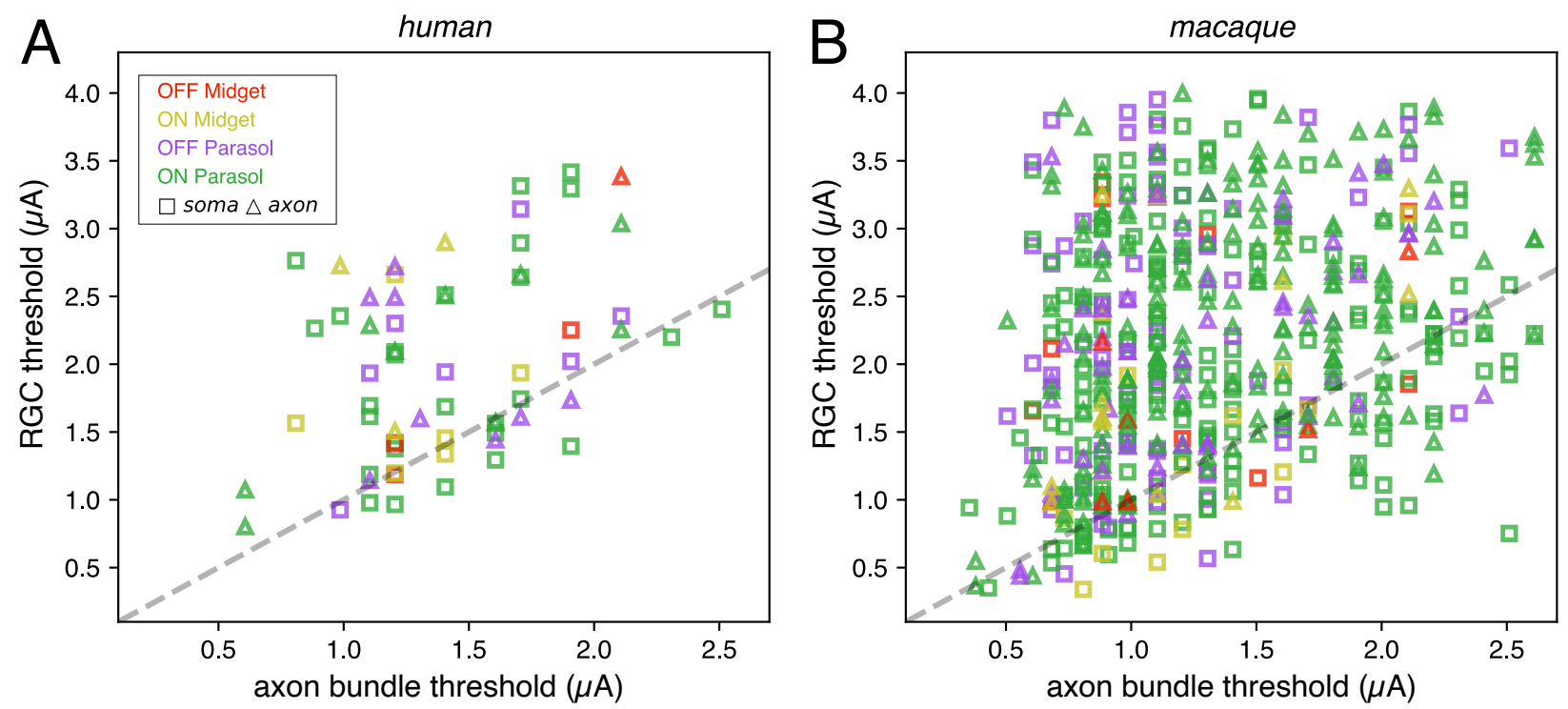

Figure 6. Single cell activation thresholds for the four major RGC types and axon bundle thresholds for the corresponding electrodes. A. Thresholds in a single human recording (43 total cells recorded in response to stimulation on 61 distinct electrodes, for a total of 66 cell-electrode pairs). Each electrode has a single associated bundle threshold, and several associated cell thresholds. B. Same as panel A, but in macaque retina (13 recordings, 1-61 cells per recording, 297 total cells and 265 distinct electrodes for a total of 313 cell-electrode pairs).
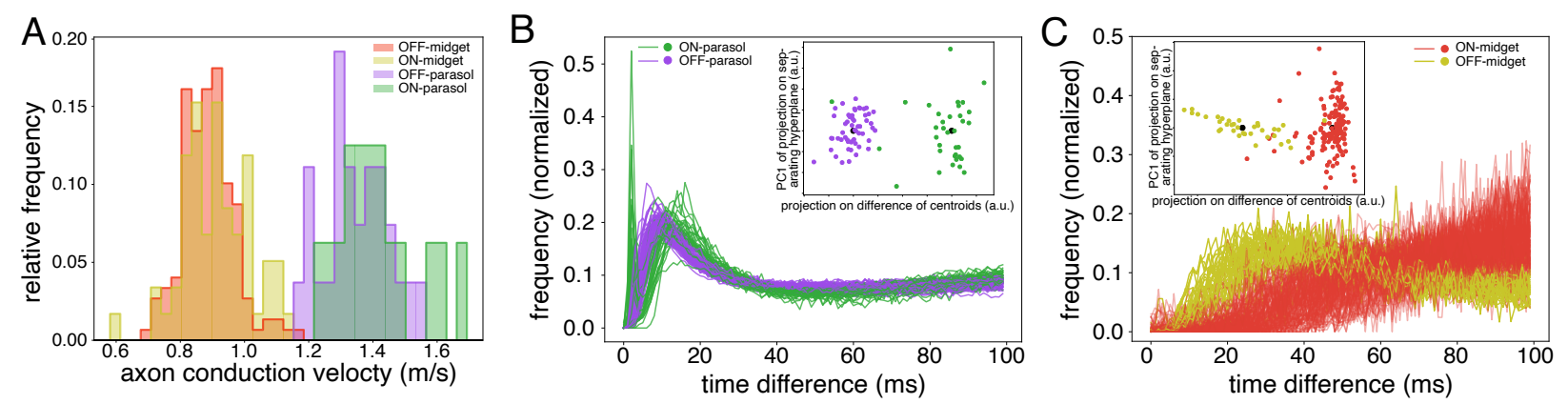

Figure 7. Cell type identification using electrical images and spike train autocorrelation functions, for the four major RGC types. A. Histograms of axon conduction velocities for $251 \mathrm{ON}$-parasol, OFF-parasol, ON-midget and OFF-midget cells computed from electrical images. B. Spike train autocorrelation functions for 89 ON and OFF parasol cells. Inset: clustering of projections onto the top 7 principal components separates ON from OFF cells. Black circles represent centroids of each cluster. C. Same as panel $\mathrm{B}$, but for $184 \mathrm{ON}$ and OFF midget cells. The same recording was used for panels $\mathrm{A}$ and $\mathrm{B}$, but $\mathrm{a}$ separate recording was used for panel $\mathrm{C}$.

Effectively utilizing high-resolution electrical stimulation in a clinical implant requires identification and targeting of functionally distinct RGC types without measuring light-evoked responses. In the macaque retina, cell type identification using only electrical properties has been demonstrated [8]. To test whether this is also possible in the human retina, electrical features were used to distinguish the four major RGC types. Axon conduction velocity, 
estimated from the Els of recorded cells (Fig. 7A, see Methods), correctly distinguished 250/251 parasol and midget cells in one recording (Fig. 7A), and 141/149 cells in a second recording (not shown) [22]. Within the parasol and midget cell classes, the autocorrelation function (ACF) of the recorded spike train was used to distinguish ON and OFF cells [14, 23, 24]. The ACFs of ON-parasol and OFF-parasol cells were visibly distinct, and $k$-means clustering $(k=2)$ of projections onto the first seven principal components enabled cell type separation that was correct for 86/89 cells in one recording (Fig. 7B) and 72/84 cells in a second recording (not shown). Discrimination of ON-midget and OFF-midget cells based on ACFs was ineffective in one recording (277/423 cells, not shown), but was effective in a second recording (176/184 cells, Fig. 7C). Although this variability requires further characterization and may pose a challenge in clinical implants (see Discussion), the results demonstrate that recorded electrical features of cells, such as Els and ACFs, have the potential to enable cell type identification.

To assess the potential application of the above findings for restoration of vision, a newly developed stimulation algorithm and assessment metric were used [11]. To produce high-fidelity artificial vision with a retinal implant, precise RGC activity evoked by individual electrodes must be combined across the array to reproduce the rich spatiotemporal response patterns evoked by natural visual stimuli. However, in general this cannot be achieved simply by passing current through multiple electrodes simultaneously, because currents delivered by different electrodes can combine nonlinearly to influence firing [7]. A recent approach uses temporally dithered current pulses [11] to address this problem, exploiting the relatively slow temporal integration of visual perception ( $20 \mathrm{~ms}$, see [25]) compared to the high temporal precision of epiretinal stimulation ( $0.1 \mathrm{~ms})$ (Fig. 2B\&E). Briefly, a target visual stimulus is converted into a sequence of single-electrode stimuli, each probabilistically evoking spikes in one or more target cells. The perceived image is assumed to depend only on the total number of spikes generated by each cell within the integration time of the visual system. This approach was simulated using measured activation curves and a linear decoding model of the resulting perceived image, with a sequence of stimuli chosen to reduce the error maximally at each time step. This procedure resulted in an expected mean-squared error of $37 \%$ relative to the random checkerboard target images. This was substantially smaller than the error between two randomly selected target images $(170 \%)$ and only slightly larger than a lower bound on the optimal algorithm (36\%; see Methods for details). The similarity of these results to previous findings in macaque retina [11] suggests that this approach may translate effectively for vision restoration in humans.

\section{Discussion}

The results of this study reveal many similarities between electrical activation of RGCs in the human and macaque retina, providing support for the macaque as an animal model for retinal implant design. As demonstrated in a companion paper [15], the four numerically dominant RGC types in the human retina -- ON-parasol, OFF-parasol, ON-midget and OFF-midget (Fig. 1) -can be recorded and identified based on their light response properties. Electrical activation of these four cell types, with sub-millisecond precision and low activation thresholds, was similar to results in macaque (Flg. 2, 4; [6]). In some cases, activation of a single RGC without activating neighboring RGCs (Fig. 5; [6]) or axon bundles (Fig. 6; [9]) was possible, providing evidence for cellular-level control of the neural code. It was also possible to distinguish these four cell types using only electrical features (Fig. 7; [8]) rather than light response properties, which would not 
be available in a blind person. Finally, simulation of a temporal dithering strategy for electrical stimulation had similar performance to previous results in macaque [11]. Below, we discuss the applicability of the present results to a future retinal implant, as well as caveats and potential extensions.

The results suggest that future retinal implants could exploit focal RGC stimulation to provide high-fidelity artificial vision to the blind. Existing implants that directly stimulate RGCs are capable of eliciting coarse light percepts, but provide little restoration of useful vision $[1,2,26]$. A major limitation of these devices is that their large stimulating electrodes result in indiscriminate activation of multiple cells and cell types. A faithful replication of the neural code of the retina requires the ability to stimulate individual RGCs of each major type in distinct, naturalistic temporal patterns. Previous ex vivo work in the macaque retina $[5,6,9]$ and the present results collectively demonstrate the possibility of such control over RGC activation, in some cases with single-cell, single-spike precision.

Axon bundles pose a major challenge for accurately reproducing the neural code: only a minority of targeted RGCs (Fig. 6A) could be activated without activating axon bundles, as in macaque retina (Fig. 6B), and consistent with perceptual reports from people with retinal implants [27]. Previous work in macaque retina showed that nearly half of the cells that could be activated without neighbors could also be activated without axon bundles [9]. Although this proportion was difficult to estimate reliably in the human data because of the small number of analyzable cells, the present findings are qualitatively consistent with the macaque findings, because cells with activation thresholds lower than those of their neighbors also tend to have thresholds lower than those of axon bundles. As in the macaque data, the findings suggest that many electrodes will not be able to avoid activating axon bundles, and therefore that a calibration procedure to identify and avoid those electrodes will be crucial for a clinical implant. Other retinal locations such as the raphe region, which contains a lower density of axon bundles compared to peripheral regions, may be important for future study in the human: results in macaque indicate that a larger fraction of RGCs in the raphe can be activated without axon bundles [9].

High-fidelity visual reconstruction relies on cell type identification, but the light response properties that can be used for this purpose in a healthy retina (Fig. 1; [15]) are unavailable in the retina of a blind person. Previous work in macaque retina [8] and the present work (Fig. 7) show that features of the electrical image (EI) and the spike train autocorrelation function (ACF) can be used to distinguish the four numerically dominant cell types, without relying on light responses. One feature of the $\mathrm{EI}$-- axon conduction velocity -- reliably separated parasol cells from midget cells (Fig. 7A) consistent with well-known biophysical distinctions between these cell types in the macaque retina $[22,28]$. Features of the ACF separated the ON and OFF types within the parasol and midget cell classes (Fig. 7B,C). However, because the temporal structure of ACFs varies between retinas, these features may be difficult to use for identifying (rather than merely distinguishing) cell types (see [14, 23]). This suggests that further analysis of El features, or patient feedback about the perceived brightness of phosphenes evoked by electrical stimulation, could be important for identifying cell types in the clinic. In addition, ON and OFF midget cells were reliably separated based on ACFs in one recording but not in the other (see Results), again motivating further investigation of El features. Finally, cells of other types were not considered in this analysis, but may be important for the function of a clinical implant. 
Changes in the retina during degeneration could introduce additional challenges. First, the electrical activity used here for cell type identification was light-driven, whereas an implant in a blind person would rely on recording spontaneous activity. Spontaneous activity in the major RGC types in anesthetized macaques is high (20-30 Hz) [29], but has not been measured in humans. Some studies have indicated that spontaneous RGC activity increases during retinal degeneration [30-32], however, results in a rat model of photoreceptor degeneration indicate that spontaneous activity increases in OFF cells but declines in ON cells [33]. Second, it is unclear whether the structure of the ACF changes during degeneration. It seems unlikely that ON and OFF cell ACFs would become much more similar to one another, unless the distinctions between them in the healthy retina are primarily a result of presynaptic activity that is lost during degeneration, which is unlikely based on previous work [33]. However, changes during degeneration could make it difficult to identify (as opposed to distinguish) cell types based on ACFs. Third, it is unclear how Els would be affected by degeneration, although their overall form probably depends mostly on the morphological structure and ion channel distribution of the cell, which may remain relatively stable (but see [34, 35]). Finally, for clinical application it will be critical to verify that electrical stimulation thresholds in degenerated retina are comparable to those in the healthy retina, as has been previously demonstrated in a rat model of retinal degeneration [36].

Caveats about the extent of the present data are also worth highlighting. First, analysis of electrical responses was restricted to a single human retinal preparation. Although comparison to the macaque revealed many similarities, the variability across retinas is unknown, so the present results should be interpreted with caution. Second, analysis of electrical responses was restricted to RGCs with large, easily detectable spikes. Only a fraction of cells identified during visual stimulation (particularly midget cells) were confirmed to be activated on at least one electrode, and a majority of cell-electrode pairs were excluded from analysis because of the difficulty of unambiguous spike identification (see Methods). Additional experiments will therefore be valuable for determining the reliability of the approaches explored here.

The present results are compatible with algorithmically combining the activation of individual RGCs over space and time to reproduce the complex spatiotemporal patterns of activity that occur in natural vision. Prior work in macaque has shown that spatiotemporal patterns of activity in small groups of cells can be reproduced with high fidelity [10]. More recently, closed-loop methods have been developed to minimize the difference between a target visual image and an estimate of the perceived image obtained through electrical activation, based on pre-calibrating the activation of RGCs of different types and temporal dithering during stimulation [11]. The performance of this method resembled results in macaque (see Results), suggesting that it can be applied to the human retina in a future implant.

Overall, the present results reveal a striking similarity in the electrical activation properties of human and macaque RGCs, and support the use of the macaque as an animal model for developing future high-fidelity retinal implants for vision restoration. 


\section{Methods}

\section{Retinas and preparation}

A human eye was obtained from a brain-dead donor (29 year-old Hispanic male) through Donor Network West (San Ramon, CA). Macaque eyes were obtained from terminally anesthetized macaques sacrificed by other laboratories, in accordance with Institutional Animal Care and Use Committee guidelines. Following enucleation the eyes were hemisected, the vitreous was removed in room light, and the eye cup was stored in oxygenated Ames' solution (Sigma) at $33^{\circ} \mathrm{C}$. Electrophysiological data were recorded from portions of the retina, as described previously $[6,9]$. Small $(\sim 3 \times 3 \mathrm{~mm})$ segments of peripheral retina $(14 \mathrm{~mm}$ from the fovea in the superior nasal quadrant) were isolated from the sclera, detached from the pigment epithelium, and held RGC side down on a multielectrode array. A second human retina recording (Fig. $7 A \& B)$ was also obtained, in which the pigment epithelium remained attached $(12 \mathrm{~mm}$ from the fovea on the superior vertical meridian). For macaque preparations, the retina was detached from the pigment epithelium and eccentricities ranged from 8 to $12 \mathrm{~mm}$ temporal equivalent eccentricity.

\section{Electrophysiological recording and visual stimulation}

Recording and stimulation were performed with a custom 512-electrode $(10 \mu \mathrm{m}$ diameter, $60 \mu \mathrm{m}$ pitch, $1.7 \mathrm{~mm}^{2}$ ) system sampling at $20 \mathrm{kHz}$, as described previously [9, 37-39]. Spikes from individual neurons were identified and segregated using standard spike sorting techniques [37, $40,41]$. To identify the type of each recorded cell, the retina was visually stimulated with a binary white noise stimulus, and the spike-triggered average (STA) stimulus was computed for each RGC $[12,13]$. The STA summarizes the spatial, temporal, and chromatic structure of the light response. The spatial receptive fields and time courses obtained from the STA were used to identify the distinct cell types (Fig. 1), as described previously [13-15, 23]. Electrical images (Els), obtained by averaging thousands of spatiotemporal voltage patterns over the array at the times of the identified spikes from each RGC $[19,37]$ were used to infer the spatial location of the soma and axon (Fig. 3) and to identify electrically elicited spikes (see below) [6]. The peak of visually and electrically evoked spikes was defined as the maximum negative recorded voltage deflection of the recorded waveform.

\section{Electrical stimulation and spike sorting}

Electrical stimuli were triphasic current waveforms, consisting of a negative stimulating phase lasting $50 \mu \mathrm{s}$ (one sample) preceded and followed by charge-balancing phases of equal duration, with relative amplitudes 2:-3:1 (Fig. 2A\&D, top inset), passed through one electrode at a time. At each electrode, forty current amplitudes in the range 0.1 to $4.1 \mu \mathrm{A}$ were delivered twenty-five times each [6, 9].

A semi-automated method was used to subtract electrical artifacts from the raw data and assign spikes to cells, as described previously [6, 9]. For each analyzed cell, spike probabilities were computed across the twenty-five repeats of each stimulus, and were modeled as a function of current amplitude by a sigmoidal relationship:

$$
p(a)=\frac{1}{1+e^{-k(a-b)}}
$$


where $a$ is the current amplitude, $p(a)$ is the spike probability, and $k$ and $b$ are free parameters (representing slope and threshold, respectively). Fitted curves were used to compute the activation threshold, defined as the current amplitude producing a spike probability of 0.5 .

To enable accurate spike assignment, only cells with recorded absolute spike amplitudes of at least $\sim 60 \mu \mathrm{V}$ were analyzed, as spikes with lower amplitudes were difficult to visually distinguish from noise. Even after filtering cells by this threshold, in some cases large electrical stimulation artifacts, axon bundle activation, or other biological activity made it difficult to assign spikes to identified cells with confidence. These cells were also excluded from further analysis. Of the 316 cells identified during visual stimulation, 43 were analyzable by the above criteria (ON-parasol: 21 analyzable / 49 identified, OFF-parasol: 10/14, ON-midget: 8/149, OFF-midget: 4/104). Among cell-electrode pairs with analyzable spikes, a large fraction (334/400) were excluded from analysis because bundle activation or other noise sources prevented unambiguous spike assignment.

\section{Estimation of axon bundle activation thresholds}

For each stimulating electrode, axon bundle activation thresholds were estimated using a method previously described [21]. Bundle activation can be detected by bidirectional propagation of an evoked electrical signal that, unlike the activity of a single cell, increases progressively in amplitude with increasing current [9]. Observers visually inspected a movie of recorded voltage on the array for a period of $2 \mathrm{~ms}$ after electrical stimulation. Bundle activation threshold was defined as the lowest stimulus amplitude at which bidirectional activity that increased with stimulating current reached at least two edges of the array, implying that the signal originated at least partly in the axons of cells with somas off the array. For a small number of electrodes near the edges of the array, bidirectional activity was not easily discerned. For bundles near these electrodes, the activation threshold was defined as the lowest current level at which unidirectional activity that grew in amplitude with current level and was clearly larger than the signal of a single axon $(\sim 35 \mu \mathrm{V})$ propagated to a single distant edge of the array.

\section{Distinguishing midget and parasol cells using axon conduction velocity}

The axon conduction velocity of each cell was estimated using axonal El waveforms upsampled tenfold to improve spike peak time estimation (Fig. 3, top; see [19, 22]). Among all the electrodes recording axonal signals from a given cell, the distance between each pair of electrodes was divided by the corresponding time difference of the negative peaks of the El waveforms, producing a pairwise velocity estimate. The conduction velocity for the cell was estimated by computing a weighted average of these pairwise estimates, with the weight for each pair of electrodes given by the product of the peak El amplitudes on the electrodes. Electrode pairs were excluded if the difference in time at minimum voltage was less than the sampling interval $(0.05 \mathrm{~ms})$. Only velocity estimates corresponding to the largest ten weight values for each cell were used. Els that were visibly corrupted by errors in spike sorting or that contained too few (less than eight) axonal electrodes were excluded from analysis. After this filtering, 251 of the total 520 Els remained in one recording and 149 of the total 270 Els remained in a second recording.

\section{Distinguishing ON and OFF cells using ACFs}

The autocorrelation function (ACF) of the recorded spike times for each cell was used to discriminate ON and OFF cells (see [14, 23]). For each cell class (midget, parasol) principal 
components analysis was performed on the normalized (L2) ACFs of all cells, and $k$-means clustering $(k=2)$ was performed on the projections onto the top seven principal components. A cell was considered correctly separated if it was assigned to a cluster in which its type was the most numerous.

\section{Analysis of temporal dithering for optimizing artificial vision}

The temporal dithering algorithm uses the measured responses to electrical stimulation to select a sequence of stimuli intended to produce perception matching a target visual image as closely as possible [11]. This approach is based on the assumption that visual perception is determined by the total number of spikes from each RGC within the integration time of the visual system ( $20 \mathrm{~ms}$; see [25]), during which many stimuli can be applied. Specifically, the approach requires a dictionary containing elements representing the spike probabilities of all cells for each distinct electrical stimulus tested (in this case, each electrode and current level), a target visual image, and a decoder which computes an estimate of the visual image from the spike counts of all cells during the integration time window. Responses of ON and OFF parasol cells that could be stimulated by at least one electrode were used to construct the dictionary. Due to the limited number of analyzable cells, dictionary elements that activated axon bundles were permitted in the analysis, although this is not appropriate for a clinical implant. Random checkerboard images were chosen as targets for reconstruction, and the scaled spatial receptive field of each cell was chosen as the linear decoding filter for its spikes (see [42]). To simulate the temporal dithering approach, a sequence of electrodes and stimulating current amplitudes was chosen greedily, maximizing the expected reduction in mean squared error between the target and linearly decoded image in each stimulation time step. Algorithm performance was measured as relative mean squared error (mean squared error divided by mean squared intensity of the target image) over the pixels covered by the receptive fields of all cells used. The error of the algorithm was compared to a lower bound on the error of an optimal algorithm, in which the entire stimulation sequence was optimized to minimize the error between the target and decoded image [11].

\section{References}

1. Humayun MS, Dorn JD, da Cruz L, Dagnelie G, Sahel J-A, Stanga PE, Cideciyan AV, Duncan JL, Eliott D, Filley E, Ho AC, Santos A, Safran AB, Arditi A, Del Priore LV, Greenberg RJ, Argus II Study Group (2012) Interim results from the international trial of Second Sight's visual prosthesis. Ophthalmology 119:779-788

2. Goetz GA, Palanker DV (2016) Electronic approaches to restoration of sight. Rep Prog Phys 79:096701

3. Stingl K, Bartz-Schmidt KU, Besch D, Braun A, Bruckmann A, Gekeler F, Greppmaier U, Hipp S, Hörtdörfer G, Kernstock C, Koitschev A, Kusnyerik A, Sachs H, Schatz A, Stingl KT, Peters T, Wilhelm B, Zrenner E (2013) Artificial vision with wirelessly powered subretinal electronic implant alpha-IMS. Proceedings of the Royal Society B: Biological Sciences 280:20130077

4. Palanker D, Le Mer Y, Mohand-Said S, Muqit M, Sahel JA (2020) Photovoltaic Restoration of Central Vision in Atrophic Age-Related Macular Degeneration. Ophthalmology 127:1097-1104

5. Sekirnjak C, Hottowy P, Sher A, Dabrowski W, Litke AM, Chichilnisky EJ (2008) High-resolution electrical stimulation of primate retina for epiretinal implant design. J Neurosci 28:4446-4456 
6. Jepson LH, Hottowy P, Mathieson K, Gunning DE, Dabrowski W, Litke AM, Chichilnisky EJ (2013) Focal Electrical Stimulation of Major Ganglion Cell Types in the Primate Retina for the Design of Visual Prostheses. Journal of Neuroscience 33:7194-7205

7. Jepson LH, Hottowy P, Mathieson K, Gunning DE, Dąbrowski W, Litke AM, Chichilnisky EJ (2014) Spatially patterned electrical stimulation to enhance resolution of retinal prostheses. J Neurosci $34: 4871-4881$

8. Richard E, Goetz GA, Chichilnisky EJ (2015) Recognizing retinal ganglion cells in the dark. In: Advances in Neural Information Processing Systems 28. Curran Associates, Inc., pp 2476-2484

9. Grosberg LE, Ganesan K, Goetz GA, Madugula SS, Bhaskhar N, Fan V, Li P, Hottowy P, Dabrowski W, Sher A, Litke AM, Mitra S, Chichilnisky EJ (2017) Activation of ganglion cells and axon bundles using epiretinal electrical stimulation. J Neurophysiol 118:1457-1471

10. Jepson LH, Hottowy P, Weiner GA, Dabrowski W, Litke AM, Chichilnisky EJ (2014) High-Fidelity Reproduction of Spatiotemporal Visual Signals for Retinal Prosthesis. Neuron 83:87-92

11. Shah NP, Madugula S, Grosberg L, Mena G, Tandon P, Hottowy P, Sher A, Litke A, Mitra S, Chichilnisky EJ (2019) Optimization of Electrical Stimulation for a High-Fidelity Artificial Retina. 2019 9th International IEEE/EMBS Conference on Neural Engineering (NER)

12. Chichilnisky EJ (2001) A simple white noise analysis of neuronal light responses. Network 12:199213

13. Chichilnisky EJ, Kalmar RS (2002) Functional Asymmetries in ON and OFF Ganglion Cells of Primate Retina. The Journal of Neuroscience 22:2737-2747

14. Field GD, Sher A, Gauthier JL, Greschner M, Shlens J, Litke AM, Chichilnisky EJ (2007) Spatial properties and functional organization of small bistratified ganglion cells in primate retina. $\mathrm{J}$ Neurosci 27:13261-13272

15. Kling A, Gogliettino AR, Shah NP, Wu EG, Brackbill N, Sher A, Litke A, Silva RA, Chichilnisky EJ (2020) Functional Organization of Midget and Parasol Ganglion Cells in the Human Retina

16. Dacey DM (2004) Origins of perception: retinal ganglion cell diversity and the creation of parallel visual pathways. In: The Cognitive Neurosciences. MIT Press, Cambridge, pp 281-301

17. Rodieck RW (1998) The first steps in seeing. Sinauer, Sunderland, MA

18. Sekirnjak C, Hottowy P, Sher A, Dabrowski W, Litke AM, Chichilnisky EJ (2006) Electrical stimulation of mammalian retinal ganglion cells with multielectrode arrays. J Neurophysiol 95:3311-3327

19. Petrusca D, Grivich MI, Sher A, Field GD, Gauthier JL, Greschner M, Shlens J, Chichilnisky EJ, Litke AM (2007) Identification and characterization of a Y-like primate retinal ganglion cell type. J Neurosci 27:11019-11027

20. Kuffler SW (1953) Discharge patterns and functional organization of mammalian retina. J Neurophysiol 16:37-68

21. Tandon P, Bhaskar N, Ganesan K, Grosberg L, Hottowy P, Dabrowski W, Sher A, Litke A, Chichilnisky EJ, Mitra S (2017) Automatic Identification and Avoidance of Axon Bundle Activation for Epiretinal Prosthesis. In: The Eye and the Chip (TEATC)

22. Li PH, Gauthier JL, Schiff M, Sher A, Ahn D, Field GD, Greschner M, Callaway EM, Litke AM, Chichilnisky EJ (2015) Anatomical identification of extracellularly recorded cells in large-scale multielectrode recordings. J Neurosci 35:4663-4675 
23. Rhoades CE, Shah NP, Manookin MB, Brackbill N, Kling A, Goetz G, Sher A, Litke AM, Chichilnisky EJ (2019) Unusual Physiological Properties of Smooth Monostratified Ganglion Cell Types in Primate Retina. Neuron 103:658-672.e6

24. Devries SH, Baylor DA (1997) Mosaic arrangement of ganglion cell receptive fields in rabbit retina. J Neurophysiol 78:2048-2060

25. Wandell BA (1995) Foundations of Vision. Oxford University Press, Incorporated

26. Weiland JD, Cho AK, Humayun MS (2011) Retinal prostheses: current clinical results and future needs. Ophthalmology 118:2227-2237

27. Nanduri $D$ (2011) Prosthetic vision in blind human patients: predicting the percepts of epiretinal stimulation. PhD, University of Southern California

28. Fukuda Y, Watanabe M, Wakakuwa K, Sawai H, Morigiwa K (1988) Intraretinal axons of ganglion cells in the Japanese monkey (Macaca fuscata): conduction velocity and diameter distribution. Neurosci Res 6:53-71

29. Troy JB, Lee BB (1994) Steady discharges of macaque retinal ganglion cells. Vis Neurosci 11:111118

30. Margolis DJ, Newkirk G, Euler T, Detwiler PB (2008) Functional stability of retinal ganglion cells after degeneration-induced changes in synaptic input. J Neurosci 28:6526-6536

31. Pu M, Xu L, Zhang H (2006) Visual response properties of retinal ganglion cells in the royal college of surgeons dystrophic rat. Invest Ophthalmol Vis Sci 47:3579-3585

32. Margolis DJ, Detwiler PB (2011) Cellular origin of spontaneous ganglion cell spike activity in animal models of retinitis pigmentosa. J Ophthalmol 2011: . https://doi.org/10.1155/2011/507037

33. Sekirnjak C, Jepson LH, Hottowy P, Sher A, Dabrowski W, Litke AM, Chichilnisky EJ (2011) Changes in physiological properties of rat ganglion cells during retinal degeneration. J Neurophysiol 105:2560-2571

34. Chen Z, Song Y, Yao J, Weng C, Yin ZQ (2013) Alterations of sodium and potassium channels of RGCs in RCS rat with the development of retinal degeneration. J Mol Neurosci 51:976-985

35. Walston S, Lee E-J, Humayun M, Weiland J (2013) Comparison of Ankyrin-G Labeling of Axon Initial Segments on Ganglion Cells in Normal and Degenerate Rat Retina. Invest Ophthalmol Vis Sci 54:1055-1055

36. Sekirnjak C, Hulse C, Jepson LH, Hottowy P, Sher A, Dabrowski W, Litke AM, Chichilnisky EJ (2009) Loss of responses to visual but not electrical stimulation in ganglion cells of rats with severe photoreceptor degeneration. J Neurophysiol 102:3260-3269

37. Litke AM, Bezayiff N, Chichilnisky EJ, Cunningham W, Dabrowski W, Grillo AA, Grivich M, Grybos P, Hottowy P, Kachiguine S, Kalmar RS, Mathieson K, Petrusca D, Rahman M, Sher A (2004) What does the eye tell the brain?: Development of a system for the large-scale recording of retinal output activity. IEEE Transactions on Nuclear Science 51:1434-1440

38. Hottowy P, Dąbrowski W, Skoczeń A, Wiącek P (2008) An integrated multichannel waveform generator for large-scale spatio-temporal stimulation of neural tissue. Analog Integrated Circuits and Signal Processing 55:239-248

39. Hottowy P, Skoczeń A, Gunning DE, Kachiguine S, Mathieson K, Sher A, Wiącek P, Litke AM, Dąbrowski W (2012) Properties and application of a multichannel integrated circuit for low-artifact, patterned electrical stimulation of neural tissue. Journal of Neural Engineering 9:066005 
40. Lee J, Mitelut C, Shokri H, Kinsella I, Dethe N, Wu S, Li K, Reyes EB, Turcu D, Batty E, Kim YJ, Brackbill N, Kling A, Goetz G, Chichilnisky EJ, Carlson D, Paninski L YASS: Yet Another Spike Sorter applied to large-scale multi-electrode array recordings in primate retina

41. Pachitariu M, Steinmetz NA, Kadir SN, Carandini M, Harris KD Fast and accurate spike sorting of high-channel count probes with KiloSort. In: Advances in neural information processing systems

42. Rieke F, Warland D, de Ruyter van Steveninck RR, Bialek W (1997) Spikes: exploring the neural code. MIT Press, Cambridge, MA 